\title{
Herlyn-Werner-Wunderlich Syndrome: A Case of Report and Literature Review
}

\author{
Huijuan Lu, Cunjian Yi ${ }^{*}$ \\ Department of Obstetrics and Gynecology, The First Affiliated Hospital of Yangtze University, Jingzhou, China \\ Email: *cunjiany@163.com
}

How to cite this paper: $\mathrm{Lu}, \mathrm{H}$.J. and $\mathrm{Yi}$, C.J. (2021) Herlyn-Werner-Wunderlich Syndrome: A Case of Report and Literature Review. Case Reports in Clinical Medicine, 10, 365-372.

https://doi.org/10.4236/crcm.2021.1011045

Received: July 23, 2021

Accepted: October 29, 2021

Published: November 1, 2021

Copyright $\odot 2021$ by author(s) and Scientific Research Publishing Inc. This work is licensed under the Creative Commons Attribution International License (CC BY 4.0).

http://creativecommons.org/licenses/by/4.0/ (c) (i) Open Access

\begin{abstract}
Background: Herlyn-Werner-Wunderlich syndrome (HWWS) is a rare type of female reproduction tract malformation, often accompanied by abnormal development of the ipsilateral urinary system, which shows mostly double uterus and the absence of the kidney on the oblique side. Case Report: An 11-year-old patient presented with lower abdominal distension and pain for 2 days and was suspected of vaginal haematoma. Conclusion: Ipsilateral renal agenesis was confirmed by ultrasound during hospitalization, and the diagnoses of obstructed hemivagina and ipsilateral renal anomaly syndrome were established. The aim of this study was to present the diagnostic and therapeutic approaches in HWWS, and to draw attention to the challenges in its diagnosis, which can coexist with multiple complications.
\end{abstract}

\section{Keywords}

Herlyn-Werner-Wunderlich Syndrome, Deformity, Diagnosis, Treatment

\section{Introduction}

Herlyn-Werner-Wunderlich syndrome (HWWS; also known as Oblique vaginal septum syndrome, OVSS) is a kind of asymmetric genitourinary congenital malformations, including didelphys uterus, unilateral blind hemivagina, and ipsilateral renal agenesis, such as absence of one side of the kidney, which is more common in HWWS, heterotopic single kidney malformation, horseshoe kidney, etc. [1] [2]. Due to non-specific clinical manifestations, the rate of missed diagnosis and misdiagnosis is extremely high [3]. A case of HWWS patient admitted to the First Affiliated Hospital of Yangtze University, the First People's Hospital of Jingzhou is reported, combined with literature review, in order to strengthen clinicians' understanding of this syndrome, improve the diagnosis rate of HWWS, and improve the prognosis of patients. 


\section{Case Presentation}

The patient was a 11-year-old unmarried woman with no history of sexual activity, and had menstrual cramps in January 2021. The first three menstrual cycles after menstruation are regular, but menstrual volume in the recent 2 months is less, and there is no dysmenorrhea. She experienced lower abdominal distension and paroxysmal pain, aggravated when cold and occasionally aggravated sensation during defecation since two days ago. Her pain did not radiate and was not associated with chills, fever, nausea, vomiting, abdominal pain, diarrhea and other discomforts. The symptoms of lower abdomen distension are significantly relieved than before, and she has not complained of any special discomfort, she came to our hospital for treatment. Gynecological examination showed that the vulva development was normal, and a purple-blue spherical mass of about $2 \times 3$ $\mathrm{cm}$ in size was visible at the vaginal opening. The tension was slightly high, and internal consultation was not performed. Transabdominal sonography showed that the uterine morphology is abnormal, and two uterine echoes can be seen in the cross section. The left uterus has a transverse diameter of $36 \mathrm{~mm}$, an anteroposterior diameter of $35 \mathrm{~mm}$, and a longitudinal diameter of $56 \mathrm{~mm}$. The right uterus has a transverse diameter of $35 \mathrm{~mm}$, an anteroposterior diameter of 29 $\mathrm{mm}$, and a longitudinal diameter of $47 \mathrm{~mm}$. The endometrium is in the middle, with a clear display and a thickness of $8 \mathrm{~mm}$. The parenchyma of the muscle layer has uniform echoes, and no obvious abnormal echoes are seen. A hypoechoic area of $136 \times 61 \mathrm{~mm}$ can be seen below the uterus, which seems to continue with the lower part of the left uterus. There was no obvious echo of fluid area in the pelvic cavity. A $50 \times 30 \mathrm{~mm}$ hypoechoic zone can be seen in the right appendage area. The boundary is still clear and there is no obvious blood flow signal. Ultrasonic diagnosis: abnormal uterine morphology: double uterus? hypoechoic area in the left uterine vagina, cervix, and uterine cavity may be blood accumulation; hypoechoic area in the right appendage area may be blood accumulation (Figure 1). Color Doppler ultrasound of the urinary system revealed that no renal echo was detected in the left kidney area (Figure 2). Clinical diagnosis: abdominal pain to be checked; vaginal oblique septum syndrome? Solitary kidney. Subsequently, the patient underwent oblique vaginal septum resection and hysteroscopy surgery. During the operation, there is a $5 \times 4 \mathrm{~cm}$ purple-blue spherical mass protruding outside the left vaginal opening, with extremely high tension, and it can be seen that the hymen edge has been cracked. One cervix can be seen in the endoscopic dilation of the right vaginal opening, $1 \mathrm{~cm}$ is cut at the left side of the tumor, and approximately $800 \mathrm{ml}$ of dark blood was evacuated. After the endoscopic dilation, another cervix can be seen. The oblique septum comes from under the double cervix and runs diagonally to the left. The left vaginal opening was closed, the mediastinum was incised with forceps from the outside to the inside, the edge of the mediastinum was sutured to stop bleeding, and the vagina was filled with two iodoform gauze. Postoperative diagnosis: left oblique vaginal septum type I, isolated kidney. Postoperative infection 


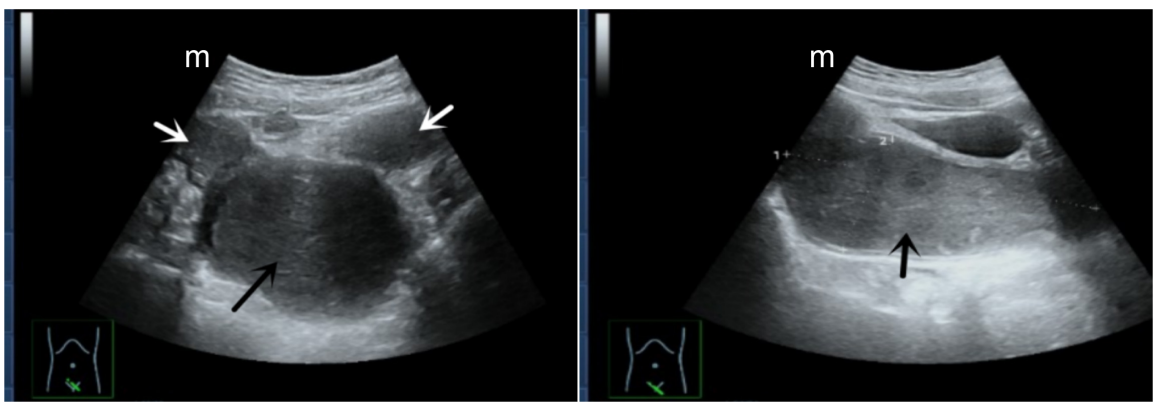

Figure 1. The sagittal plane of the patient's gynecological pelvic ultrasound showed two uterine bodies (white arrow) and vaginal hemorrhage (black arrow).

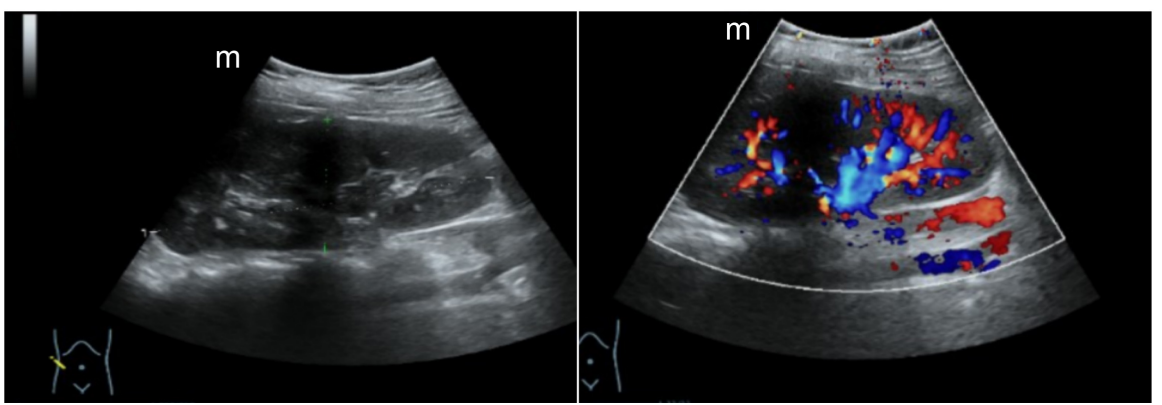

Figure 2. The ultrasound of the patient's urinary system showed that the right kidney was visible.

prevention, fluid rehydration, and symptomatic treatment are given. Oblique incision through the vagina, the symptoms of the patient disappeared quickly, and the prognosis was good. She has recovered well and has been discharged from hospital.

\section{Discussion}

\subsection{Pathogenesis}

The incidence of HWWS is $0.1 \%-3.8 \%$, and currently the pathogenesis is unclear [4]. The vagina is formed by the development and connection of the end of the accessory mesonephric duct and the urogenital sinus. On the embryonic side, the mesonephric duct and the accessory mesonephric duct are underdeveloped. They are not in contact with the urogenital sinus and cannot form an unobstructed vagina. The accessory mesonephros fusion fails, and the twin uterus, twin cervix and vaginal septum malformations develop respectively [5] [6]. The oblique vaginal septum is a membranous tissue that grows down from between the two cervixes, forming a blind cavity on one side obliquely to cover the cervix on this side. Current research shows that the mechanism of the asymmetric deformity of the oblique vaginal septum is still unclear, which may be related to the downward extension of the accessory mesonephric duct without contacting the urogenital sinus and the formation of a blind end [7] [8]. The urinary system and the reproductive system have a common origin. The development of the accessory mesonephric duct depends on the development of the mesoneph- 
ric duct. The dysplasia of the mesonephric duct often coexists with the dysplasia of the accessory mesonephric duct. Therefore, the malformation of the reproductive system is often accompanied by There are malformations of the urinary system. This disease is characterized by double uterus, double cervix, double vagina, complete or incomplete atresia of one side of the vagina, and often accompanied by absence of kidney and ureter on the oblique side. There are reports that the right kidney is absent, while this case is on the left side [9] [10].

\subsection{Clinical Classification}

At present, the classification of Peking Union Medical College Hospital is mostly used in China [11]. The deformity is divided into 3 types: type I (no Oblique hole type): One side of the vagina is completely atresia, the uterus behind the septum is completely isolated from the outside and the opposite uterus, there is no traffic between the two uteruses and between the two vaginas, and the uterine cavity accumulates in the vagina behind the septum; Type II (perforated oblique septum type): One side of the vagina is not completely atresia, and there are small holes about several millimeters in diameter on the septum, menstrual blood can be discharged through the small holes, but the discharge is not smooth; type III (non-porous oblique septum combined with cervix Fistula type): One side of the vagina is completely atresia, and there is a small fistula between the cervix on both sides or between the vaginal cavity of the posterior septum and the contralateral cervix. The blood accumulated in the posterior septum can be discharged through the contralateral cervix, but the discharge is not smooth. Type 3 all merged with oblique septal kidney loss. This patient is HWWS I, which is prone to misdiagnosis during clinical diagnosis. Therefore, she encounters developmental malformations of reproductive organs in clinical work. Careful physical examination before surgery and adequate exploration during surgery are required to reduce misdiagnosis [12].

\subsection{Diagnosis}

\subsubsection{Clinical Symptoms}

The clinical manifestations of HWWS: 1) Dysmenorrhea: The degree of dysmenorrhea varies, which may be the main reason of seeing a doctor for patients. 2) Prolonged menstrual period or vaginal discharge, discharge of pus: mostly manifested as menstrual dripping. 3) Vaginal wall cyst: It is the most important positive sign, but it can also cause misdiagnosis or missed diagnosis due to atypical. 4) Pelvic mass: It forms when a large amount of vaginal fluid spreads to the ipsilateral uterus or fallopian tube. 5) Absence of kidney on one side. 6) Pelvic endometriosis: rarely occurs, and may be related to long-term menstrual reflux [13]. This patient was 11 years old and had menarche. Because of her young age, the uterus, cervix, and vagina were not fully developed, so she could not perform transvaginal diagnosis. Gynecological examination was limited, and it was not easy to find positive signs in the vagina. The patient complained of pain in the 
lower abdomen, accompanied by ultrasound prompting a large amount of blood in the vagina, which is clinically very similar to hymen atresia. If only the obstruction of the lower genital tract is considered, the uterine development abnormality is missed and misdiagnosis is easy to occur. Clinically, patients with genital tract dysplasia with no menarche during adolescence and emergency treatment should be fully considered and fully evaluated before surgery. In particular, MRI can clearly diagnose vaginal oblique septum syndrome and assist in the evaluation of its clinical classification [12] [14] [15]. When a patient has one side of the kidney, especially in adolescent women, combined with genital malformations, pelvic masses, and primary dysmenorrhea, HWWS should be considered first.

\subsubsection{Auxiliary Inspection}

1) Ultrasound: It is the most simple and non-invasive method at present. It can clearly show specific changes such as uterus malformation, effusion under the cervix, and absence of kidney. Color Doppler ultrasound mostly displays images of the pelvic cavity and double uterus; both are below the oblique side of the cervix. Visible liquid dark zone or low echo zone. The menstrual blood retention showed a large dark area, full and spherical shape; this type of patients did not explore the ultrasound image of one side of the normal kidney [16]. However, the resolution of ultrasonography is low, and local details and adjacent relationships are not as clear as CT and MRI [17]. 2) CT: CT has a higher resolution than ultrasound, and can obtain clearer images. The powerful post-processing technology can obtain important information more intuitively and comprehensively, which is of great help to the diagnosis and pre-treatment evaluation of the disease. However, CT shows that the relationship between the posterior cavity effusion and the cervix is not as clear and accurate as MRI, and the display of endometrial condition is not good. CT examination has a certain degree of radiation. The patients diagnosed with HWWS are mostly children, young women, or pregnant women, there exist limitations. 3) MRI: The positive rate of diagnosis is high, and the image levels are rich. It can clearly show the relationship between the posterior cavity effusion and the cervix, and can make a preliminary judgment on the composition of the cavity effusion, which provides evidence for a correct diagnosis before surgery and the development of surgical plans [2]. 4) Hysterosalpingography: It can diagnose uterine malformations and find the location of the leak, but it is mostly used for type III, type I and type II are easy to missed diagnosed. 5) KUB-IVP: It can indicate the absence of kidney and ureter on the affected side. 6) Combined hysteroscopy and laparoscopy: it can be diagnosed and treated at the same time. Laparoscopy can clearly show the types of uterine malformations and the nature of pelvic masses, observe whether there is pelvic endometriosis, pelvic adhesions, etc., and perform corresponding surgical treatments at the same time. Hysteroscopy can understand the conditions of the vagina, cervix and uterine cavity, and can also be treated [13] [18]. 


\subsection{Differential Diagnosis}

Due to the diversity of clinical manifestations of HWWS, it is easy to be misdiagnosed. Clinically, it is easy to be confused with the following common diseases: 1) Functional uterine bleeding: In type II and III oblique septum syndrome, there is repeated irregular vaginal bleeding. 2) Pelvic inflammatory disease: When the oblique vaginal septum syndrome is repeatedly bleeding with infection, symptoms of pelvic inflammatory disease such as purulent vaginal discharge, fever, and abdominal pain may appear. 3) Pelvic mass: hemorrhage or empyema on the affected side, ectopic cyst, pelvic examination and color Doppler ultrasound can be mistaken for pelvic tumor or abscess. 4) Endometriosis: Patients with oblique vaginal septum syndrome have progressively aggravated dysmenorrhea due to poor menstrual blood drainage or menstrual blood reflux. Color Doppler ultrasound suggests that there are dot-like dense echoes in the cyst, which is often confused with endometriotic cysts. 5) Others such as ectopic pregnancy, infertility, appendicitis, residual horn uterine bleeding (or pregnancy), vaginal wall cysts, etc., should sometimes be differentiated from HWWS [19]. HWWS I patients mainly present with dysmenorrhea, which is easily misdiagnosed as primary dysmenorrhea, vaginal wall cysts, and pelvic masses. Type II and Type III patients mainly manifested as vaginal bloody or purulent discharge, which is easily misdiagnosed as vaginitis, pelvic inflammatory disease, adolescent dysfunctional uterine bleeding, etc. [20].

\subsection{Treatment}

HWWS patients are mostly adolescents and women of childbearing age. The principle of treatment is to repair and reconstruct functions based on the type of genital tract malformations, try to protect the function and integrity of the organs, and relieve the obstruction of the outflow tract. Therefore, the minimally invasive hysteroscopy combined with vaginal oblique diaphragm resection has advantages. There are three kinds of surgical methods: oblique vaginal septum resection, oblique vaginal septum resection combined with hysteroscopy and hysterectomy in the posterior cavity. Oblique vaginal septum resection is the most fundamental surgical method for the treatment of HWWS. In type III patients, the cervical fistula should be repaired at the same time during the operation [4]. After the operation, the vagina was filled with iodophor gauze for 24 to 48 hours, and antibiotics were used to prevent infection. Potassium permanganate powder was bathed for 7 to 10 days [21].

\section{Conclusion}

All in all, the diagnosis of HWWS may currently be a challenge for gynecologists. Gynecologists, pediatricians, and radiologists should consider the differential diagnosis of HWWS in genital tract abnormalities to improve the diagnosis rate of HWWS. The uterus on the oblique side of the vagina may still be pregnant, and resection should be avoided as much as possible to improve the pa- 
tient's ability to conceive. Whether to remove the uterus on the oblique side of the vagina is still controversial. There are few follow-up cases of postoperative pregnancy in HWWS patients, and further samples need to be accumulated for analysis and summary. At present, the mechanism of the asymmetric deformity of the oblique vaginal septum is not fully understood, and further research is needed.

\section{Ethical Approval and Consent to Participate}

This work follows the guidelines for human studies and was conducted ethically in accordance with the World Medical Association Declaration of Helsinki. Written informed consent was obtained from the patient for reporting and publication of this case report and any accompanying images and the relevant approval was obtained from the ethical committee of the First Affiliated Hospital of Yangtze University.

\section{Consent for Publication}

The consent for publication was obtained from both the patient and the First Affiliated Hospital of Yangtze University.

\section{Availability of Supporting Data}

The data is stored in the electronic database of the First Affiliated Hospital of Yangtze University under the confidentiality act.

\section{Author Contributions}

H.J.L. developed the concept, collected the data, and wrote the manuscript. C.J.Y. participated in writing the manuscript. C.J.Y. analysed the data and coordinated efforts. All authors contributed to editorial changes in the manuscript. All authors read and approved the final manuscript.

\section{Conflicts of Interest}

The authors declare no conflict of interest.

\section{References}

[1] Chowdhury, S., Ara, R., Begum, S.A., et al. (2015) Uterus Didelphys with Unilateral Obstructed Hemivagina and Haematocolpos with Ipsilateral Renal Agenesis-A Case Report. Mymensingh Medical Journal, 24, 202-206.

[2] He, A. and Feng, P.Y. (2017) A Case of Oblique Vaginal Septum Syndrome and Literature Review. Chinese Journal of Clinical Medical Imaging, 28, 75-76.

[3] Tang, H.M. and Wang, L.F. (2003) Analysis of 9 Cases of Oblique Vaginal Septum Syndrome. Chinese Journal of Practical Gynecology and Obstetrics, No. 4, 17.

[4] Wdowiarz, K., Skrajna, A. and Reinholz-Jaskólska, M. (2021) Diagnosis and Treatment of Herlyn-Werner-Wunderlich Syndrome: A Case Report. Menopause Review, 20, 52-56. https://doi.org/10.5114/pm.2021.104034

[5] Liang, H.I., et al. (2020) Herlyn-Werner-Wünderlich Syndrome: An Unusual Case 
with Presentation of Menorrhagia. Taiwanese Journal of Obstetrics and Gynecology, 59, 948-951. https://doi.org/10.1016/j.tjog.2020.09.026

[6] Cao, Z.Y. (2014) Chinese Obstetrics and Gynecology. Chinese Clinicians, 42, 95.

[7] Noviello, C., Romano, M., Nino, F., Martino, A. and Cobellis, G. (2018) Clinical and Radiological Findings for Early Diagnosis of Herlyn-Werner-Wunderlich Syndrome in Pediatric Age: Experience of a Single Center. Gynecological Endocrinology, 34, 56-58. https://doi.org/10.1080/09513590.2017.1332178

[8] Wang, J.H., Zhu, L., Lang, J.H., et al. (2005) Clinical Analysis of Oblique Vaginal Septum Syndrome. Advances in Modern Obstetrics and Gynecology, No. 5, 72-73. https://doi.org/10.13283/j.cnki.xdfckjz.2005.05.019

[9] Tuna, T., et al. (2019) Herlyn-Werner-Wunderlich Syndrome: Report of a Prenatally Recognised Case and Review of the Literature. Urology, 125, 205-209. https://doi.org/10.1016/j.urology.2018.12.022

[10] Zhang, J. (2011) A Case of Oblique Vaginal Septum Syndrome and Literature Review. Jiangxi Medicine, 46, 1141-1142.

[11] Acién, P. and Acién, M. (2016) The Presentation and Management of Complex Female Genital Malformations. Human Reproduction Update, 22, 48-69. https://doi.org/10.1093/humupd/dmv048

[12] Wang, W.L., Zhang, T. and Ye, H. (2020) Three Cases of Misdiagnosis of Oblique Vagina Syndrome and Literature Review. Modern Medicine, 48, 1466-1469.

[13] Li, Y.F. and Liu, Y.H. (2012) A Case of Oblique Vaginal Septum Syndrome. Shandong Medicine, 52, 40-41.

[14] Dias, J.L. and Jogo, R. (2015) Herlyn-Werner-Wunderlich Syndrome: Pre- and Post-Surgical MRI and US Findings. Abdominal Imaging, 40, 2667-2682. https://doi.org/10.1007/s00261-015-0421-0

[15] Wang, Y., Lin, Q., Sun, Z.J., et al. (2018) Application Value of MRI in the Preoperative Diagnosis and Clinical Classification of Oblique Vaginal Septum Syndrome. Chinese Journal of Obstetrics and Gynecology, 53, 534-539.

[16] Chen, J. and Wang, H.J. (2019) A Case Report of Oblique Vaginal Septum Syndrome. Journal of Sichuan University (Medical Edition), 50, 33.

[17] Li, H.D. and Wu, Z.Y. (2011) Ultrasound Diagnosis of Congenital Oblique Vaginal Septum Syndrome. International Journal of Obstetrics and Gynecology, 38, 247-249.

[18] Li, X., Liu, T. and Li, L. (2021) Herlyn-Werner-Wunderlich Syndrome and Its Complications: A Report of Two Cases and Literature Review. Radiology Case Reports, 16, 2319-2324. https://doi.org/10.1016/j.radcr.2021.05.055

[19] Qiu, Y.F. (2013) A Case of Oblique Vaginal Septum Syndrome and Analysis of Diagnosis and Treatment. Chinese Medicine Guide, 11, 622-623.

[20] Zhu, S.N. and Zhu, Y.J. (2017) Diagnosis and Treatment of Oblique Vaginal Septum Syndrome. International Journal of Obstetrics and Gynecology, 44, 262-264.

[21] Fu, C., Zhang, H.W. and Fang, X.L. (2011) Clinical Analysis of 28 Cases of Oblique Vaginal Septum Syndrome. Journal of Central South University (Medical Edition). 36, 809-812. 